\title{
Improvement of Peak Cough Flow After the Application of a Mechanical In-exsufflator in Patients With Neuromuscular Disease and Pneumonia: A Pilot Study
}

\author{
Ji Ho Jung, MD , Hyeon Jun Oh, MD', Jang Woo Lee, MD², Mi Ri Suh, MD ${ }^{1,3}$, Jihyun Park, MD ${ }^{1}$, \\ Won Ah Choi, $\mathrm{MD}, \mathrm{PhD}^{1,3}$, Seong-Woong Kang, $\mathrm{MD}, \mathrm{PhD}^{1,3}$
}

${ }^{1}$ Department of Rehabilitation Medicine and Rehabilitation Institute of Neuromuscular Disease, Gangnam Severance Hospital, Yonsei University College of Medicine, Seoul;

${ }^{2}$ Department of Physical Medicine and Rehabilitation, National Health Insurance Service Ilsan Hospital, Goyang;

${ }^{3}$ Pulmonary Rehabilitation Center, Gangnam Severance Hospital, Yonsei University College of Medicine, Seoul, Korea

Objective To investigate and demonstrate persistent increase of peak cough flow after mechanical in-exsufflator application, in patients with neuromuscular diseases and pneumonia.

Methods A mechanical in-exsufflator was applied with patients in an upright or semi-upright sitting position (pressure setting, +40 and $-40 \mathrm{cmH}_{2} \mathrm{O}$; in-exsufflation times, 2-3 and 1-2 seconds, respectively). Patients underwent five cycles, with 20-30 second intervals to prevent hyperventilation. Peak cough flow without and with assistive maneuvers, was evaluated before, and 15 and 45 minutes after mechanical in-exsufflator application.

Results Peak cough flow was $92.6 \mathrm{~L} / \mathrm{min}$ at baseline, and 100.4 and $100.7 \mathrm{~L} / \mathrm{min}$ at 15 and $45 \mathrm{minutes}$ after mechanical in-exsufflator application, respectively. Assisted peak cough flow at baseline, 15 minutes, and 45 minutes after mechanical in-exsufflator application was $170.7,179.3$, and $184.1 \mathrm{~L} / \mathrm{min}$, respectively. While peak cough flow and assisted peak cough flow increased significantly at 15 minutes after mechanical in-exsufflator application compared with baseline ( $\mathrm{p}=0.030$ and $\mathrm{p}=0.016)$, no statistical difference was observed between 15 and 45 minutes.

Conclusion Increased peak cough flow after mechanical in-exsufflator application persists for at least 45 minutes.

Keywords Mechanical in-exsufflator, Neuromuscular diseases, Pneumonia, Peak cough flow

Received May 21, 2018; Accepted June 26, 2018

Corresponding author: Seong-Woong Kang

Department of Rehabilitation Medicine, Gangnam Severance Hospital, 211 Eonju-ro, Gangnam-gu, Seoul 06273, Korea. Tel: +82-2-2019-3492, Fax: +82-2-2019-3499, E-mail: kswoong@yuhs.ac

ORCID: Ji Ho Jung (http://orcid.org/0000-0002-3597-2227); Hyeon Jun Oh (http://orcid.org/0000-0001-8235-5435); Jang Woo Lee (http://orcid. org/0000-0002-2634-0375); Mi Ri Suh (http://orcid.org/0000-0003-1759-9198); Jihyun Park (http://orcid.org/0000-0002-2172-1072); Won Ah Choi (http://orcid.org/0000-0003-0403-8869); Seong-Woong Kang (http://orcid.org/0000-0002-7279-3893).

(c) This is an open-access article distributed under the terms of the Creative Commons Attribution Non-Commercial License (http://creativecommons.org/ licenses/by-nc/4.0) which permits unrestricted noncommercial use, distribution, and reproduction in any medium, provided the original work is properly cited. Copyright ( 2018 by Korean Academy of Rehabilitation Medicine 


\section{INTRODUCTION}

Respiratory infection is the most common cause of hospitalization, in patients with neuromuscular diseases [1]. Especially, in patients with amyotrophic lateral sclerosis (ALS), respiratory complications due to excessive airway secretions and ineffective coughing, are principal causes of morbidity and mortality [2-4]. Among patients with Duchenne muscular dystrophy, $90 \%$ die of respiratory complications [5-7]. Thus, it is important to effectively remove airway secretions in patients with neuromuscular diseases, who have respiratory muscle weakness.

Effectiveness of the removal of airway secretions, depends on peak cough flow (PCF). According to previous studies, a PCF value of at least $160 \mathrm{~L} / \mathrm{min}$ is needed for effective removal of airway secretions $[1,8,9]$.

A mechanical in-exsufflator (MI-E) is used in patients with neuromuscular disease, with reduced PCF to remove airway secretions. By alternately applying positive and negative pressure to the airway, MI-E is used to remove secretions in the airway. This can be effectively used for removal of secretions in patients with respiratory infections with excessive sputum, as well as in patients with neuromuscular diseases showing decreased ability to voluntarily cough [10].

Previous studies have shown that PCF increases, during or immediately after MI-E application, in patients with neuromuscular diseases [10-13]. However, it is unknown whether increased PCF persists for a certain period, after application of MI-E. Therefore, in addition to investigating effects of MI-E, this study examined changes in PCF before and after a certain period, from application of MI-E in patients with neuromuscular diseases who have pneumonia and difficulty in voluntarily expelling sputum because of weakened respiratory muscles.

\section{MATERIALS AND METHODS}

\section{Subjects}

This study was conducted on subjects with neuromuscular diseases with pneumonia admitted to a university hospital from August 2015 to July 2016. Pneumonia was diagnosed based on clinical symptoms, and laboratory and radiologic findings. Evidence of cardinal infection such as fever or chills and leukocytosis, signs or symptoms localized to the respiratory system (cough, increased sputum production, and increased respiratory rates), and a new or changed infiltrate on simple radiography were analyzed. All patients enrolled in the study received intravenous antibiotics therapy, for treatment of pneumonia, and the study was conducted during treatment.

The following patients were excluded: those with a risk for barotrauma and a history of pneumothorax or lung bullae; those in whom PCF could not be checked because of severe bulbar dysfunction; those who need continuous ventilator support, and could not perform the test; and those who showed unstable vital signs. We did not exclude pediatric patients; however, we excluded patients with impaired cognitive function.

\section{Study protocol}

We applied the MI-E to patients according to protocol of our institute, based on cumulative experiences and other previous research. During the application, patients were in an upright or semi-upright sitting position. Settings of the MI-E were $+40 \mathrm{cmH}_{2} \mathrm{O}$ and $-40 \mathrm{cmH}_{2} \mathrm{O}$ pressure with insufflation and exsufflation times of 2-3 and 1-2 seconds, respectively [13]. Patients underwent a total of five cycles, with a pause of 20-30 seconds between each cycle to prevent hyperventilation [14].

PCF without assistance and assistive PCF (APCF) were evaluated sequentially as follow: PCF and APCF before MI-E application, PCF and APCF 15 minutes after MI-E, and PCF and APCF 45 minutes after MI-E. Non-assisted PCF was measured by asking the patient to inhale maximum amount of air unassisted, before coughing as hard as possible. APCF was measured by first asking the patient to inhale maximum amount of air unassisted, breathing in additional amount of air through a mask by using a manual resuscitator bag, and then an assistant applied a strong push to the patient's abdomen while the patient was coughing [10]. Each process was repeated at least three times, and maximum value was selected from measurements (Fig. 1). Protocol was approved by the Institutional Review Board of Gangnam Severance Hospital (No. 3-2015-0149).

Statistical analysis was performed using IBM SPSS statistics version 20 (IBM, Armonk, NY, USA). Repeatedmeasures analysis of variance was used to compare values of PCF and APCF at each time, with Bonferroni correction for post hoc analysis. 
(A)

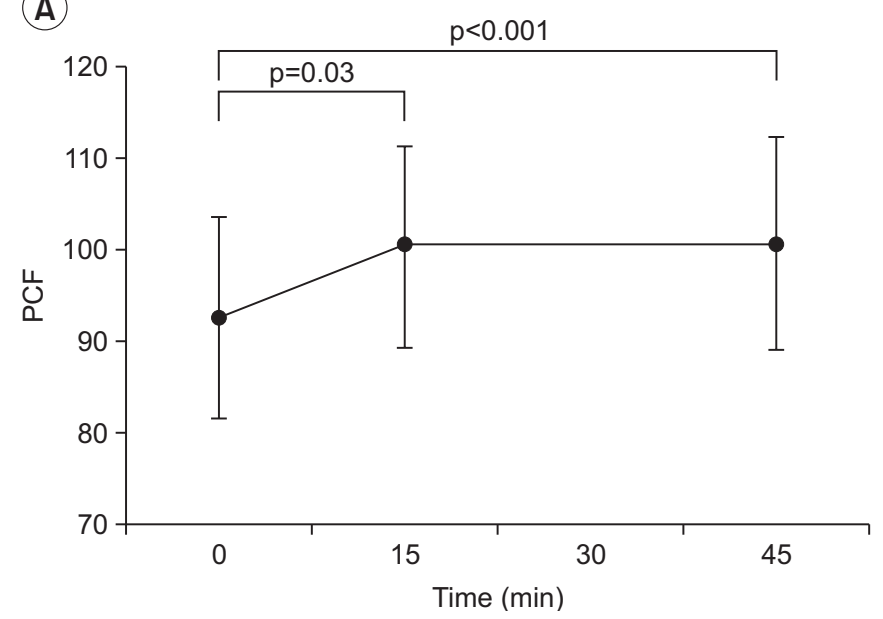

(B)

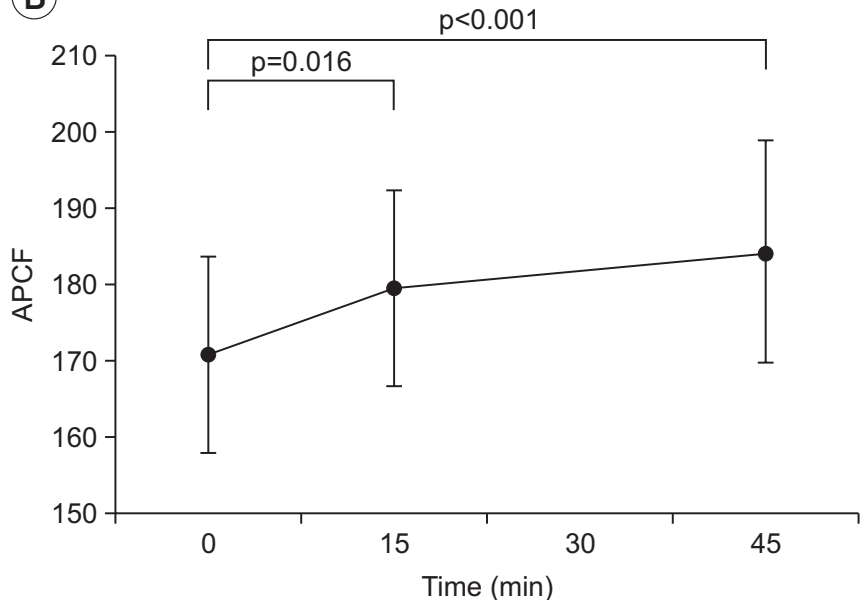

Fig. 1. (A) PCF and (B) APCF before and after mechanical in-exsufflator application. Each graph shows the mean (dot) and standard error (bar). PCF, peak cough flow; APCF, assisted peak cough flow.

Table 2. Measured PCF according to time

\begin{tabular}{|c|c|c|c|c|c|c|}
\hline & \multirow{2}{*}{ Before } & \multirow{2}{*}{15 min } & \multirow{2}{*}{$45 \mathrm{~min}$} & \multirow{2}{*}{ p-value ${ }^{a)}$} & \multicolumn{2}{|c|}{ Post-hoc analysis (p-value) } \\
\hline & & & & & Before vs. $15 \mathrm{~min}$ & $15 \mathrm{~min}$ vs. $45 \mathrm{~min}$ \\
\hline $\mathrm{PCF}(\mathrm{L} / \mathrm{min})$ & $92.6 \pm 11.0$ & $100.4 \pm 11.0$ & $100.7 \pm 11.7$ & $<0.001$ & 0.030 & $>0.999$ \\
\hline $\operatorname{APCF}(\mathrm{L} / \mathrm{min})$ & $170.7 \pm 12.7$ & $179.3 \pm 12.9$ & $184.1 \pm 14.5$ & $<0.001$ & 0.016 & 0.158 \\
\hline
\end{tabular}

Values are presented as mean \pm standard error.

PCF, peak cough flow; APCF, assisted peak cough flow.

${ }^{\text {a) }}$ Repeated-measure ANOVA.

\section{RESULTS}

A total of 27 patients (11 with Duchenne muscular dystrophy, 10 with progressive muscular dystrophy, 3 with ALS, 2 with spinal muscular atrophy, and 1 with limbgirdle muscular dystrophy) were included. Mean age was $30.0 \pm 13.3$ years (range, 12-66 years), and 21 of them (77.8\%) were men. All patients had a functional disability and could not walk without support. Twenty-four of them were using non-invasive ventilator support, and none of them was using invasive ventilator support (Table 1).

In baseline evaluation before application of MI-E, initial PCF value was $92.6 \pm 57.1 \mathrm{~L} / \mathrm{min}$ and the APCF value was $170.7 \pm 66.0 \mathrm{~L} / \mathrm{min}$. At 15 minutes after application of MI-E, measured PCF was $100.4 \pm 57.2 \mathrm{~L} / \mathrm{min}$ and the APCF was $179.3 \pm 12.4 \mathrm{~L} / \mathrm{min}$. At 45 minutes later, PCF was $100.7 \pm 60.9 \mathrm{~L} / \mathrm{min}$ and the APCF was $184.1 \pm 75.5 \mathrm{~L} / \mathrm{min}$. The increase in PCF and that in APCF compared with their baseline values, were both statistically significant $(p=0.001)$. Comparing baseline PCF and APCF with mea-
Table 1. Baseline characteristics of patients

\begin{tabular}{lc|}
\hline \multicolumn{1}{c}{ Characteristic } & Value \\
\hline Age $(\mathrm{yr})$ & $30.0 \pm 13.3$ \\
\hline Sex & \\
\hline Male & 21 \\
\hline Female & 6 \\
\hline Use of non-invasive ventilator & $24(82.8)$ \\
\hline Respiratory parameters & \\
\hline FVC $(\mathrm{mL})$ & $652.4 \pm 296.6$ \\
\hline FVC $(\%$ of predicted value $)$ & $16.6 \pm 8.9$ \\
\hline MIP $\left(\mathrm{cmH}_{2} \mathrm{O}\right)$ & $16.6 \pm 9.4$ \\
\hline MIP $(\%$ of predicted value $)$ & $19.7 \pm 13.4$ \\
\hline MEP $\left(\mathrm{cmH}_{2} \mathrm{O}\right)$ & $19.2 \pm 12.7$ \\
\hline MEP $(\%$ of predicted value $)$ & $16.3 \pm 13.5$ \\
\hline
\end{tabular}

Values are presented as mean \pm standard deviation or number (\%).

FVC, forced vital capacity; MIP, maximal inspiratory pressure; MEP, maximal expiratory pressure. 
sured value after 15 minutes, a statistically significant difference was found for both parameters $(\mathrm{p}=0.03, \mathrm{p}=0.016)$. However, comparing PCF and APCF values measured after 15 minutes with those measured after 45 minutes, peak flow was increased but the difference between time points was not statistically significant (Table 2, Fig. 1).

\section{DISCUSSION}

This study demonstrated that PCF and APCF increase for a certain period, after use of MI-E in patients with neuromuscular disease and pneumonia. According to previous studies, compared with unassisted coughing and other standard cough augmentation techniques (physiotherapy-assisted, non-invasive ventilator-assisted, and exsufflation-assisted coughing), the MI-E significantly increased PCF in patients with neuromuscular disease [10-13]. However, although existing studies have demonstrated increase in PCF during or immediately after use of MI-E, none of the studies showed whether the effects persist for a certain period. This study is the first to demonstrate increased PCF even after a certain period after application.

Maintaining increased cough flow is more critical in patients treated for current respiratory infection, because all of them have coughing disability and trouble with eliminating secretion. Nonetheless, whereas many previous studies usually excluded patients with pneumonia, we chose patients treated for pneumonia.

Many patients with neuromuscular disease, have respiratory muscle weakness that leads to declined lung compliance. Consistent increase in PCF may be explained as increase in compliance of the lung and chest wall. According to previous studies, lung compliance and the capacity for breathing increased for 3 hours, upon MI-E application in patients with kyphoscoliosis or ALS $[15,16]$. We can assume that the forced vital capacity and PCF, are increased temporarily by increased lung compliance. Furthermore, increased cough flow may be due to temporary conditioning, and strengthening effects on the respiratory muscle of the stretching exercise [17]. Stretching of respiratory muscles facilitates actin-myosin interaction, and strengthens respiratory muscles. Reduced airway resistance after removal of respiratory secretions, is also a probable mechanism, and has been proven in many previous studies.
This study has limitations. We assumed that the 15 minutes time point reflects the short-term effect of MI-E and 45 minutes reflect the lasting effect. However, 45 minutes is not sufficient time to reflect lasting effect of treatment. Maximum insufflation capacity, maximal expiratory pressure, and maximal inspiratory pressure were checked only at baseline, and only PCF and APCF were checked during follow-up. It is also a limitation that the mechanism of increasing PCF, was not definitively explained. A longer-termed and detailed evaluation is needed to explain lasting effect of this treatment. Severity, type, and disease course of pneumonia, as well as patient status at the moment of evaluation during the hospital stay, were not considered in detail, all of which could have an effect on response to treatment with MI-E.

In addition, the training effect may also be considered. It would be difficult to explain the consistent increase in PCF after an intervention, as all subjects were repeatedly evaluated in PCF and APCF consecutively during followup periods also. Therefore, the training effect should be considered, as repetitive instructions also increase subjects' compliance with using MI-E.

As this was not a blinded or randomized control study, we could not conclude whether increased PCF affect the course of pneumonia. The effect of increased PCF in patients with neuromuscular disease and pneumonia could have been more definitely evaluated, if another group such as patients with pneumonia not using MI-E or patients without pneumonia was compared. Further case-control studies involving a large group of subjects and more detailed evaluation, are needed to explain the accurate mechanism for these effects.

In conclusion, increased ability to expectorate sputum was confirmed after using MI-E, which persisted for at least 45 minutes after therapy. MI-E is a useful device for patients with neuromuscular disease and pneumonia, not only as the best way to manage secretion, but also as an intervention to maintaining coughing ability.

\section{CONFLICT OF INTEREST}

No potential conflict of interest relevant to this article was reported. 


\section{REFERENCES}

1. Bach JR, Ishikawa Y, Kim H. Prevention of pulmonary morbidity for patients with Duchenne muscular dystrophy. Chest 1997;112:1024-8.

2. Lechtzin N, Wiener CM, Clawson L, Chaudhry V, Diette GB. Hospitalization in amyotrophic lateral sclerosis: causes, costs, and outcomes. Neurology 2001;56: 753-7.

3. Andersen PM, Borasio GD, Dengler R, Hardiman O, Kollewe K, Leigh PN, et al. EFNS task force on management of amyotrophic lateral sclerosis: guidelines for diagnosing and clinical care of patients and relatives. Eur J Neurol 2005;12:921-38.

4. Bach JR. Amyotrophic lateral sclerosis: prolongation of life by noninvasive respiratory AIDS. Chest 2002; 122:92-8.

5. Inkley SR, Oldenburg FC, Vignos PJ Jr. Pulmonary function in Duchenne muscular dystrophy related to stage of disease. Am J Med 1974;56:297-306.

6. Rideau Y, Gatin G, Bach J, Gines G. Prolongation of life in Duchenne's muscular dystrophy. Acta Neurol (Napoli) 1983;5:118-24.

7. Vignos PJ Jr. Respiratory function and pulmonary infection in Duchenne muscular dystrophy. Isr J Med Sci 1977;13:207-14.

8. Bach JR. Amyotrophic lateral sclerosis: predictors for prolongation of life by noninvasive respiratory aids. Arch Phys Med Rehabil 1995;76:828-32.

9. Bach JR. Mechanical insufflation-exsufflation. Comparison of peak expiratory flows with manually assisted and unassisted coughing techniques. Chest 1993;
104:1553-62.

10. Chatwin M, Ross E, Hart N, Nickol AH, Polkey MI, Simonds AK. Cough augmentation with mechanical insufflation/exsufflation in patients with neuromuscular weakness. Eur Respir J 2003;21:502-8.

11. Sancho J, Servera E, Díaz J, Marin J. Efficacy of mechanical insufflation-exsufflation in medically stable patients with amyotrophic lateral sclerosis. Chest 2004;125:1400-5.

12. Winck JC, Goncalves MR, Lourenco C, Viana P, Almeida J, Bach JR. Effects of mechanical insufflationexsufflation on respiratory parameters for patients with chronic airway secretion encumbrance. Chest 2004;126:774-80.

13. Fauroux B, Guillemot N, Aubertin G, Nathan N, Labit A, Clement A, et al. Physiologic benefits of mechanical insufflation-exsufflation in children with neuromuscular diseases. Chest 2008;133:161-8.

14. Bach JR. Update and perspective on noninvasive respiratory muscle aids. Part 2 . The expiratory aids. Chest 1994;105:1538-44.

15. Sinha R, Bergofsky EH. Prolonged alteration of lung mechanics in kyphoscoliosis by positive pressure hyperinflation. Am Rev Respir Dis 1972;106:47-57.

16. Lechtzin N, Shade D, Clawson L, Wiener CM. Supramaximal inflation improves lung compliance in subjects with amyotrophic lateral sclerosis. Chest 2006; 129:1322-9.

17. Chatwin M. How to use a mechanical insufflator-exsufflator "cough assist machine". Breathe 2008;4:3209. 